\title{
Multiple Concentric Node Test of Modulation and Demodulation Based on Orthogonal Transformation and MOOCS
}

\author{
Qiuyue Zhu \\ Ministry of Education, Jiangxi Institute of Fashion Technology, Nanchang, 330201, China \\ qiuyue_zhu@yeah.net
} Keywords: Multiple concentric, VP- tree model, Channel capacity, Modulation and demodulation,
Orthogonal transformation, MOOCs.

\begin{abstract}
With the rapid development of MOOCs at home and abroad, the dropout low through rate results in unsatisfactory teaching effects problem that has gradually become the focus of study. In order to improve the MOOCs platform effect, using multiple concentric learning theory designs MOOCs multi angle movable concentric learning model, using VP- model constructs the node to expand the capacity of the channel, making the learning platform channel can accommodate multi angle learning content, and using the modulation and demodulation and orthogonal transformation can get the multi channel acceptance model. Finally, through the analysis of the information transmission delay characteristic curve, we found that the virtual platform can be multi channel communication effectively by user end learning content to accept the curve, the method can effectively improve the efficiency of learning.
\end{abstract}

\section{Introduction}

MOOCs is based on the open education of connectionism theory and network learning, these courses are sequenced by difficulty, enabling the student to progress from beginner to advanced with traditional university curriculum, the scope of the course not only covers science widely, such as mathematics, statistics, computer science, natural science and engineering, but also includes social science and the humanities [1-3]. In order to study the learning effect of MOOCs learning style and improve the receiving efficiency of the diverse learning, MOOCs multiple concentric node learning model is validated by using the VP-tree model structure, in which the overall frame is shown in Figure 1.

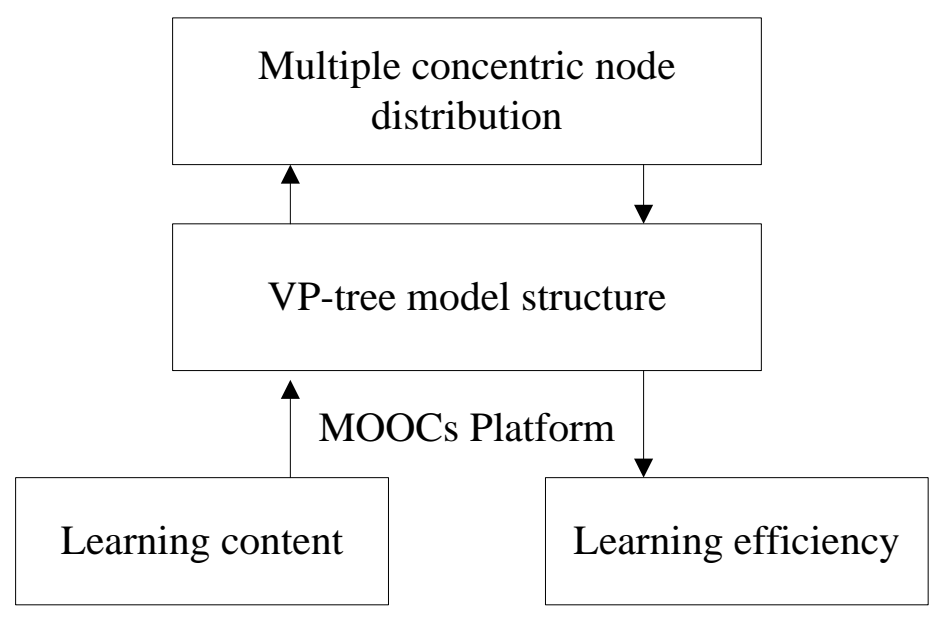

Fig.1: The multiple concentric learning frameworks

As shown in Figure 1, with the aid of the MOOCs platform, the number of nodes are constructed by using VP- model, and using multi angle communication principle designs the capacity of the channel, making learning platform channel can accommodate multi angle learning content, and then the mathematical model of the multi angle receiving signal is obtained by using the modulation and demodulation and orthogonal transformation [4,5]. Finally, through the MOOCs platform of building mobile node distribution, it can verify user end to accept the efficiency on the learning content, so as to verify the effectiveness and reliability of mathematical model. 


\section{Design of Multi Angle Study Method Modeling and Algorithm}

Multi angle learning model takes the multi channel information receiving theory as the basis, when the capacity of receiving information is greater at the same time, the effect of multi angle study will be better in theory [6-8]. In order to study the capacity calculation problems of the multi channel learning model, this paper develops a channel capacity calculation. Assuming the signal power is $P$, noise power is $q$, and then the channel capacity of disturbance continuous channel is

$$
Q=K \log _{2}\left(1+\frac{P}{q}\right)=K \log _{2}\left(1+\frac{P}{n_{0} \cdot K}\right) .
$$

Among them, $Q$ is the channel capacity, $K$ is the channel bandwidth, $P$ is the signal average power, $q$ is the noise average power, $n_{0}$ is the power spectral density of unilateral learning, $P / q q$ is the signal to noise ratio [9]. In order to improve the efficiency of the multi angle information receiving, the data is divided by using multiple reference points in a node, which to a certain extent improve the efficiency of the search as shown in Figure 2.
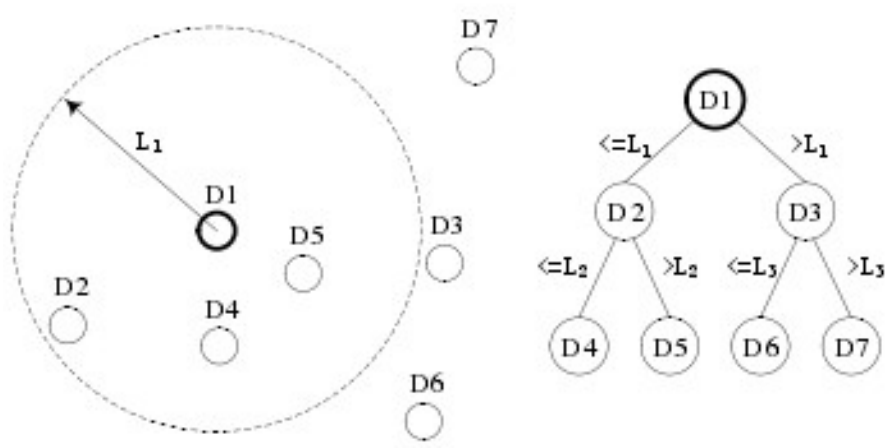

Fig.2: VP- tree multi angle model

As shown in Figure 2, the data object of reference point $a$ and $b$ will be selected by using VP- tree in each layer, and using the data builds the second tree and so on, we can obtain more trees forest, and then they are extended to the general expression.

Assuming that single frequency modulation signal is $y(t)=\cos \left(\omega_{a} t\right)$, carrier is $b(t)=\cos \left(\omega_{b} t\right)$. We can obtain the multiple angle of signal formula that is

$$
Q(t)=\cos \left(\omega_{a} t\right) \cdot \cos \left(\omega_{b} t\right)=\frac{1}{2}\left[\cos \left(\omega_{a}+\omega_{b}\right) t+\cos \left(\omega_{a}-\omega_{b}\right) t\right] .
$$

Using the VP- tree the multi angle of model, we can obtain the signal modulation formula that is

$$
\begin{aligned}
Q(t)=\cos \left(\omega_{a}+\omega_{b} t\right) & =\cos \omega_{a} t \cdot \cos \omega_{b} t-\sin \omega_{a} t \cdot \sin \omega_{b} t \\
& =\cos \omega_{a} t \cdot \cos \omega_{b} t-\cos \left(\omega_{a}-\frac{\pi}{2}\right) t \cdot \cos \left(\omega_{b}-\frac{\pi}{2}\right) .
\end{aligned}
$$

Then the learning theory model of the multi angle channel can be expressed as

$$
Q(t)=y(t) \cos \omega_{b} t \mp \hat{y}(t) \sin \omega_{b} t .
$$

Among them, $\hat{y}(t)$ is $y(t)$ Hilbert transform, it gets signal after all frequency components of $y(t)$ is all phase shifting, called $f(t)$ orthogonal signal. In order to achieve the multi band verification of the multi angle study model, this paper uses MATLAB software to carry on algorithm programming, in which the main program is as follows [10]:

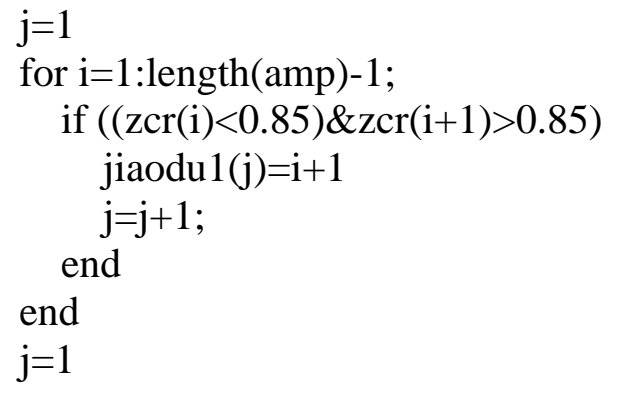




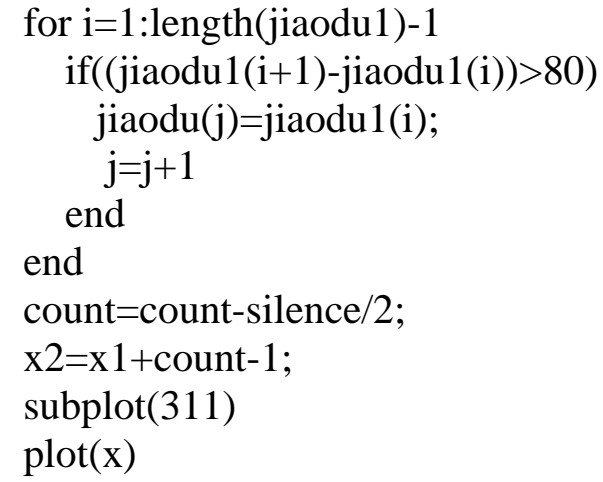

\section{The Simulation Experimental Study of MOOCs Multiple Concentric Learning Model}

In order to verify the effectiveness and reliability of multi angle concentric learning model proposed in second section, this paper builds a virtual simulation platform of diversified MOOCs learning, and obtains multi angle information receiving module by setting up the communication module processor, in which its product is shown in Figure 3.

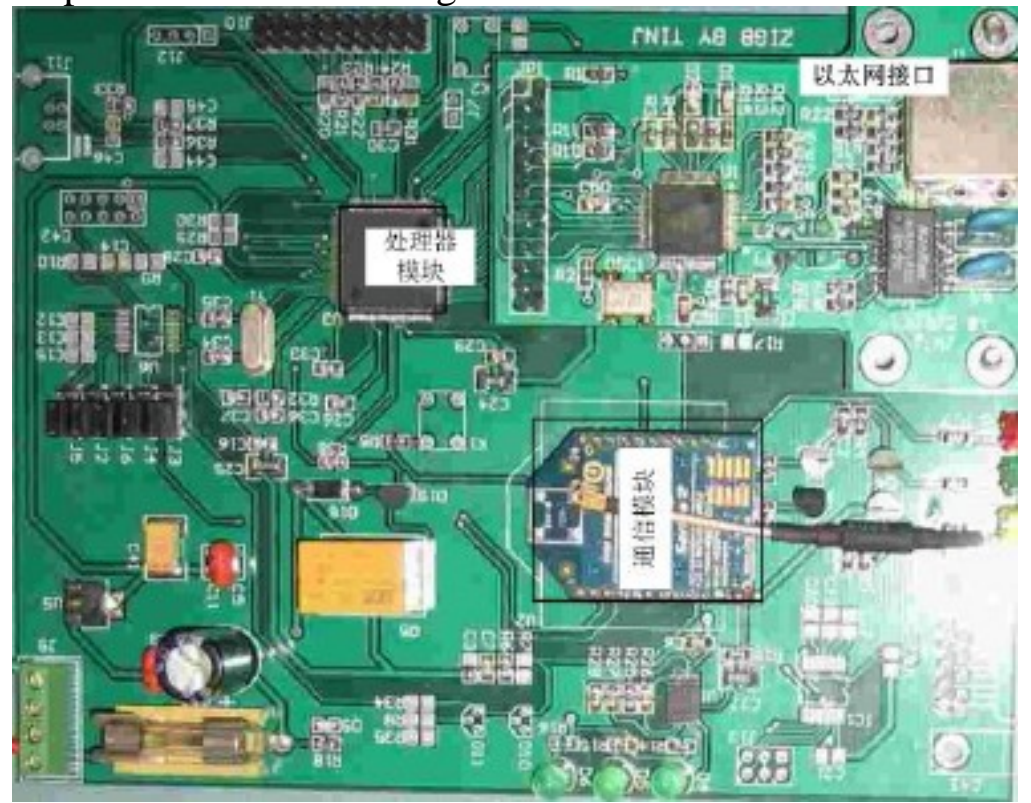

Fig.3: MOOCs multi channel design development board

Figure 3 shows the circuit expansion board physical map of receiving multi angle concentric signal, they are mainly composed of three modules, including processor module, communication module and Ethernet receiving module [11,12]. In order to realize multi angle communication channel, this paper designs the VP- tree multi angle node distribution network as shown in Figure 4.

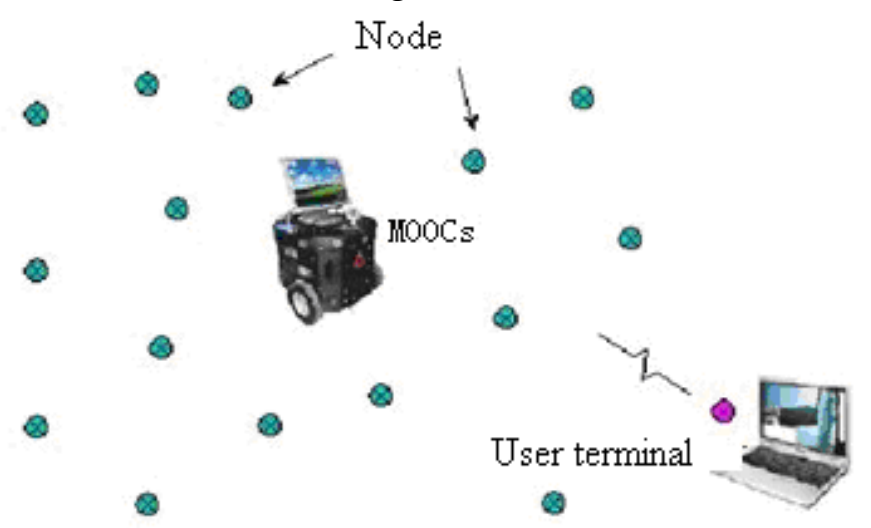

Fig.4: The multi angle VP- tree node distribution 
Figure 4 shows the multi angle VP- tree node distribution diagram, MOOCs signals receiving node is designed by the use of mobile node. Multiple concentric information will be received by using the VP-tree reference node, it can feedback to the client [13]. Finally the client can go through comprehensive study of different kind s of subjects, to comprehensive improve the learning effect.

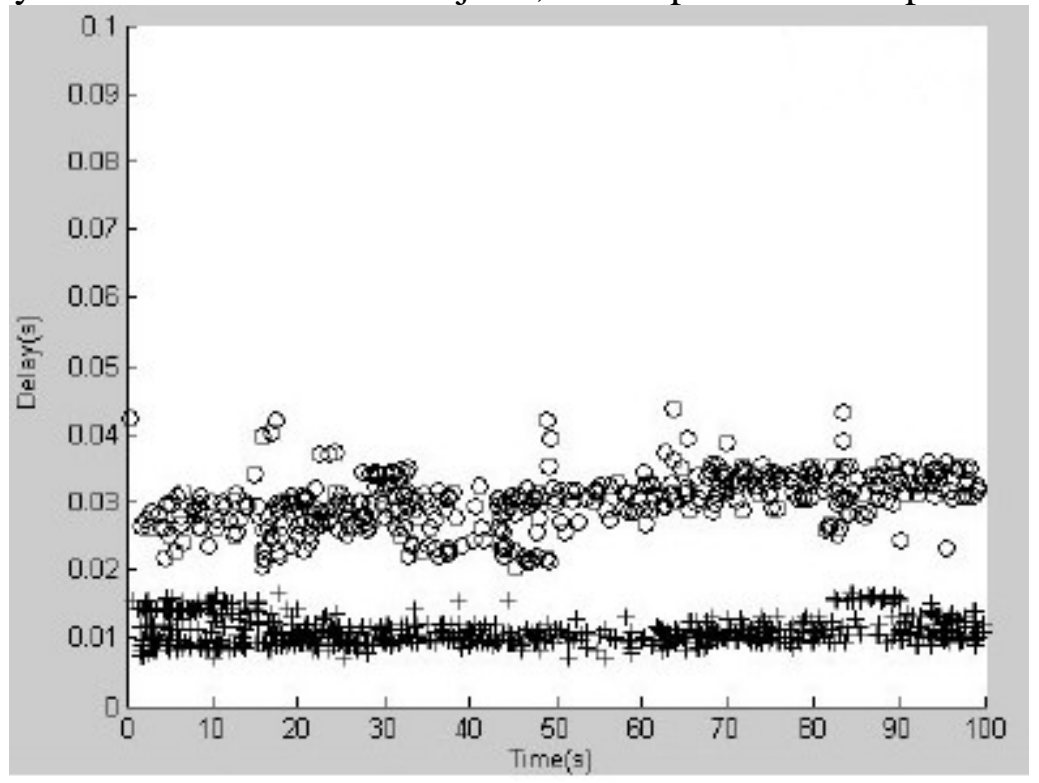

Fig.5: Single node test results

In order to verify the effect of wireless communication network communication, we first analyze the time delay characteristics of the single node that can be obtained the delay characteristic curve [14]. As shown in Figure 5, the result can be seen that the delay characteristics of single node do not exceed 5ms, and it can test on multiple nodes as shown in Figure 6.

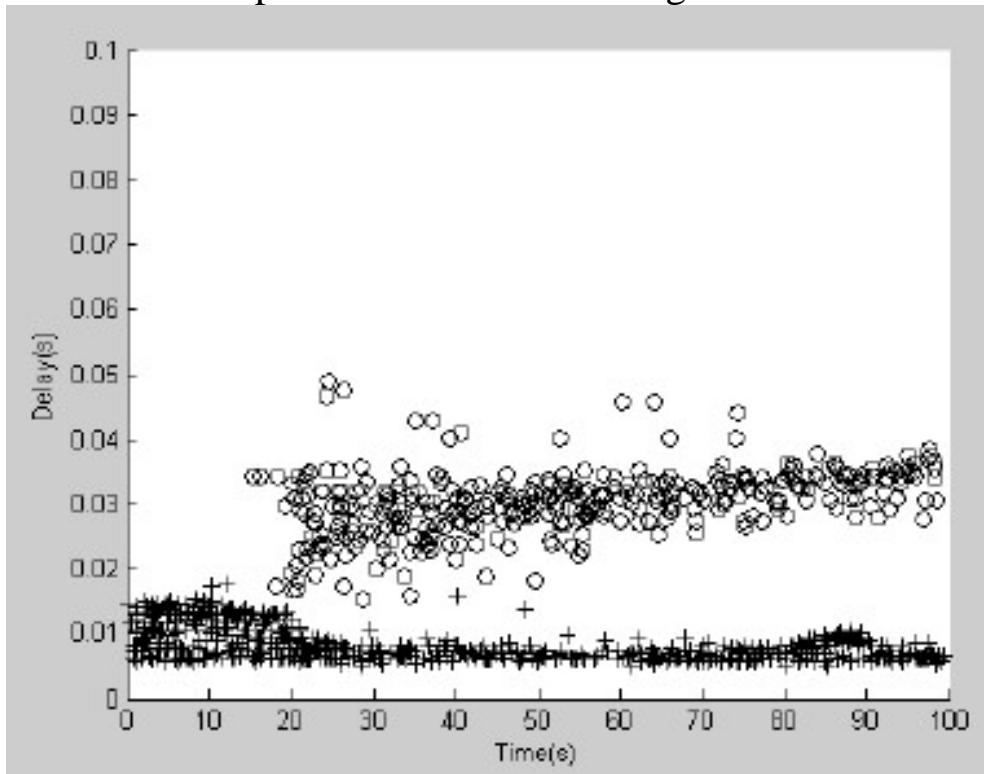

Fig.6: Multiple node test results

Figure 6 shows the time delay characteristics result curve of multiple node tests, it can be seen from the chart that the multiple nodes have little impact on the delay characteristic curve, and the maximum delay characteristics are no more than 55ms, to meet the design requirements [15]. Then we carry out statistics on the comprehensive efficiency of learning effects, the effect curve is obtained shown in Figure 7. 


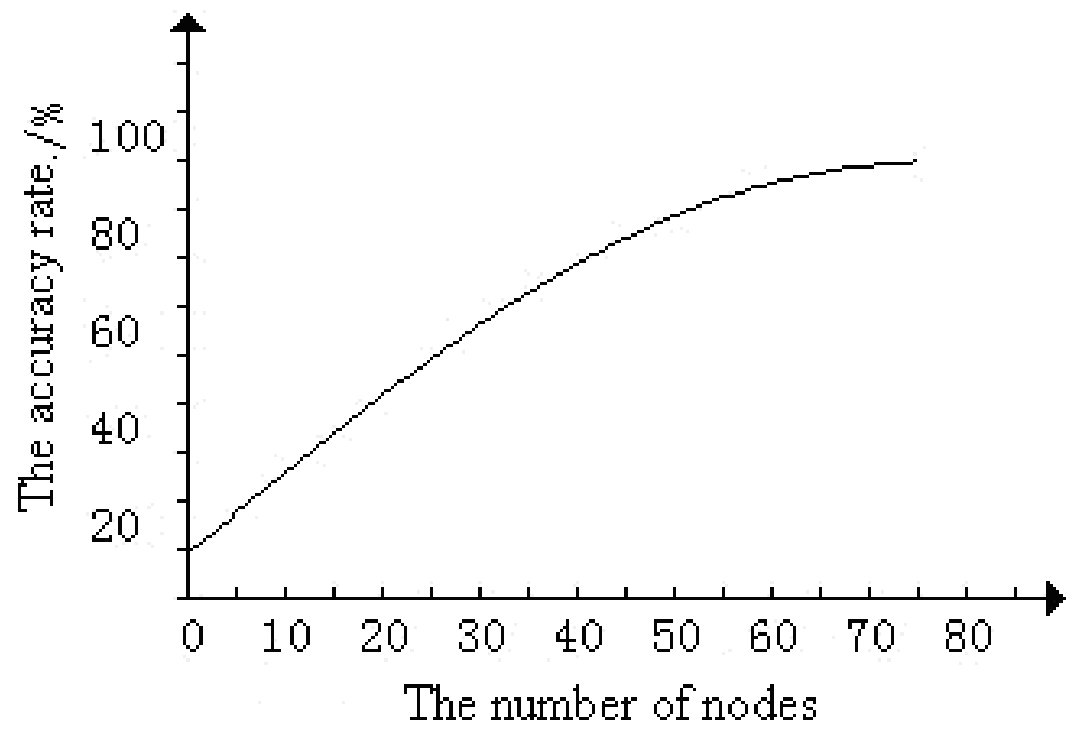

Fig.7: Multiple concentric learning effect curve

Figure 7 shows the efficiency curve of multiple concentric learning with the node number changes. With the increase of the number of nodes, learning efficiency increases; when it is added to a certain number, accepting state is at saturation, to achieve the best efficiency; when the number of nodes increase, the effect of accepting will no longer increase.

\section{Summary}

(1) Based on MOOCs learning platform, this paper designs a multiple concentric learning theory model, and the use of modulation and demodulation and orthogonal transformation principle design multi-channel acceptance model to widen the channel capacity, which improves the efficiency of data transmission.

(2) This paper designs multiple nodes and multi angle mobile information receiver platform, and multi channel delay characteristic curve are obtained by test. From the statistical curve of user terminal on the learning effect, we found that the algorithm can greatly improve the learning efficiency and broaden the learning domain, which is a reliable learning model.

\section{References}

[1] Y.Q. Kang. Online education "post MOOC era" - SPOC analysis. Tsinghua University education research, 2014(1): 24-28.

[2] J.L. Zhang, G.D. He. Analysis of learning: promoting the data online professional development of university teachers age. Journal of distance education, 2014(1): 78-81.

[3] X.B. Han, G.W. Shuang, Q. Zhou, J.G. Cheng. A comparative study on MOOC platform and its typical network teaching platform. China audio visual education, 2014(1): 34-38.

[4] X.B. Han, L.N. Cheng, J.G. Cheng. the analysis and design of MOOCs education perspective. Audio visual education studies, 2014 (1): 81-84.

[5] X.Q. Gu, Y.L Hu, H.Y. Cai. MOOCs localization appeal and its response. Distance education magazine, 2013(1): 41-42.

[6] Y.H. Wu, D. Chen, X.L. Ma. A new wave of learning education information analysis. Journal of distance education, 2013(4): 91-95.

[7] H.Q.Yu, Y. Liu, P. Li. Application of robust recursive kernel learning modeling method in blast furnace process. Journal of Zhejiang University, 2012(4): 45-48.

[8] H.Q. Wang, F.C. Sun, Y.N. Cai. Multiple kernel learning method. Acta automatica Sinica, 2012(8): 49-50.

[9] K. Chen, Y. Liu, H.Q. Wang. The adaptive algorithm of hammerstein system of recursive identification. Journal of Zhejiang University, 2010(1): 23-26. 
[10] Y. Liu, K. Chen, H.Q. Wang, P. Li. Application of selective recursive LSSVR and its process modeling. Journal of University chemical engineering, 2011(6): 34-37.

[11] Y. Liu, H.Q. Wang, P. Li. The support vector machine regression method of adaptive partial least squares in fermentation process online modeling. Journal of chemical industry and engineering, 2012(8): 12-15.

[12] Y. Liu, H.Q. Wang, P. Li. Application of the key nuclear network and its application in fermentation process online modeling. Journal of chemical industry and engineering, 2011(5): 98-99.

[13] X.B. Ma, A.X. Yan. The prediction of support vector machine used by the alkylbenzene heat capacity and standard enthalpy value. Journal of Beijing University of Chemical Technology, 2012 (2): 136-137.

[14] Y. Liu, H.Q. Wang, P. Li. Application of the local least squares support vector machine regression on-line modeling method and its batch process. Journal of chemical industry and engineering, 2011(11): 112-113.

[15] H.Q. Wang, N. Jiang. The adaptive kernel learning network classifier of process fault diagnosis. Journal of chemical industry and engineering, 2011(9): 78-80. 